\title{
Microplastics: a multidimensional contaminant requires a multidimensional framework for assessing risk
}

\author{
Kennedy Bucci ${ }^{*}$ (I) and Chelsea M. Rochman*
}

\begin{abstract}
The global ubiquity and demonstrated toxicity of microplastics has led governments around the world to express the need for a risk assessment on microplastics. To conduct a risk assessment, scientists often draw upon frameworks from other contaminants, however we argue that microplastics are a unique pollutant and thus require a unique framework. Microplastics are a multidimensional contaminant, differing in size, shape, polymer type, and chemical cocktail. Each of these dimensions may influence the toxicity of the particle. Furthermore, microplastic pollution exists as a complex and dynamic mixture of particles, that varies over temporal and spatial scales. Thus, we propose a multidimensional risk framework for microplastics that incorporates, rather than simplifies, the multidimensionality of the contaminant as well as the contaminant mixture. With this framework, we can calculate a particle-specific hazard value that describes the potential for a single particle to cause harm based on its chemical and physical properties. The particle-specific hazard values can then be combined based on the number and type of particles in an environmental sample to inform the overall hazard value of the sample. The risk of the sample can then be calculated, which is dependent on the overall hazard value and the concentration of particles in the sample. Risk values among samples in the environment can be compared to illustrate differences among locations or seasons, or can be placed in a management framework with thresholds to guide regulatory decisions. To demonstrate the utility of our proposed framework, we perform a case study using data from San Francisco Bay. Our proposed framework is just that, and requires new research for application. To strengthen the ability of this framework to accurately predict risk, we propose a testing scheme that prioritizes strategic experimental designs that will increase our understanding of how each dimension of microplastics affect the toxicity (or hazard value) of a particle.
\end{abstract}

Keywords: Plastic pollution, Microplastic, Risk assessment, Hazard, Management framework

\section{Introduction}

The global ubiquity and demonstrated toxicity of microplastics has led governments around the world to express the need for a risk assessment on microplastics, or at least a framework for performing one. Environmental or ecological risk assessments are traditionally used to evaluate the impacts of a substance on wildlife and ecosystems. They combine the hazard of the substance (its

*Correspondence: k.bucci@mail.utoronto.ca; chelsea.rochman@utoronto.ca Department of Ecology and Evolutionary Biology, University of Toronto, Toronto, ON M5S3B1, Canada potential to produce harm) and its exposure (likelihood of wildlife interacting with the substance) to calculate risk, and are used to inform management decisions. Without local risk assessments, how can governments advise on how much microplastic is too much in drinking water, fish tissue, or an aquatic ecosystem? Because there are tens of thousands of chemicals on the market [1], one might think that scientists and decision-makers could easily use lessons learned from another contaminant to inform a risk assessment framework for microplastics. Yet, it is not that simple. 
Microplastics are unlike other environmental contaminants, making it difficult to assess their toxicity. They are a multidimensional contaminant, varying in shape, size, polymer type, additive chemistry, and sorbed environmental contaminants [2]. Unlike trace metals or persistent organic pollutants, microplastics are both a chemical and a physical stressor [3]. Their chemical stress is driven by the complex suite of chemicals with which they are associated, made up of unreacted monomers, additives from manufacturing, and sorbed environmental contaminants such as persistent organic pollutants and heavy metals [4]. Their physical stress is driven by the particles themselves, which may lacerate tissue, cause inflammation, or lead to food dilution [5].

One of the barriers to conducting a risk assessment for microplastics is the difficulty in assessing their hazard, which is driven by their multidimensionality. Exercises aiming to conduct a risk assessment end up in conversations about whether to treat the contaminant as a particle or a chemical, or whether to have a different risk assessment for different shapes, sizes, polymers, etc. Some groups have developed risk assessment frameworks that simplify the dimensions of microplastics by aligning all particles in a sample to a standardized shape and size range [6-8]. In this paper, we aim to develop a framework that maintains the complexity of microplastics by capturing the hazard associated with both their physical and chemical characteristics to assess risk.

Toxicity testing to date has suggested that many sizes, types, and shapes of microplastics can be harmful to organisms $[9,10]$. Although most studies have not been designed to answer questions about how the dimensions of microplastics drive toxicity, some studies have made a concerted effort to do this. For example, Abarghouei et al. [11] exposed goldfish to $0.25 \mu \mathrm{m}$ and $8 \mu \mathrm{m}$ PS fragments and found that smaller particles induced more severe histological lesions in the liver, intestines, and gills. Polymer type has also been identified as a potential driver of toxicity, as some polymer types are made up of monomers that are inherently more toxic than others, in addition to the specific additive loads associated with each polymer type as a finished product. Lithner et al. [12] classified polymer types by the hazard of their monomers, indicating that polypropylene (PP) and polyethylene (PE) are among the least hazardous while polyvinyl chloride (PVC) and polystyrene (PS) are among the most hazardous. Empirical testing with the leachates from different plastic products confirmed these classifications, where leachates from all PVC products tested were acutely toxic to $D$. magna and only one leachate from ten PE or PP products was toxic [13]. Finally, the presence of sorbed environmental contaminants can cause microplastics to be more harmful to organisms. A study exposing Japanese medaka to microplastics with and without sorbed environmental contaminants showed exacerbated liver toxicity and pathology in the treatment where the plastic was deployed in San Diego Bay for three months [14].

The current body of microplastics toxicity literature is useful to begin building a risk assessment framework for microplastics, especially when multiple doses are used and/or experiments are designed to test how effects vary among the dimensions of microplastics. However, researchers have employed a wide variety of methodologies, and most studies are not designed to measure effect thresholds or determine whether thresholds differ among dimensions. For example, in Gray and Weinstein [15], conclusions are drawn about the effects of particle shape, despite particles being of different polymer types. These confounding variables within and amongst studies can make results difficult to interpret. Building a body of toxicity data that can be used to inform a multidimensional risk assessment framework will require studies that investigate the relative toxicity of each dimension of microplastics (i.e. shape, size, polymer type, chemical suite) while holding the other dimensions constant.

Trying to understand the risk associated with microplastics in the face of all of this complexity is daunting. Still, taking a holistic approach is warranted. In addition to asking 'does this specific microplastic cause this effect', we should also begin asking 'what characteristics of microplastics in general are driving effects, and at what concentrations?. This type of approach is relevant as strategic effects testing has suggested that spherical, additive- and environmental contaminant- free microplastics are the least harmful formulation of microplastics $[10$, 16]. A stronger database that asks these types of questions would put the field in a better position to conduct a risk assessment that accounts for the multidimensionality of microplastics.

Here, we propose a framework for evaluating the risk of microplastics as a complex, multidimensional contaminant. We have identified four dimensions that are relevant to the hazard of an individual particle and are often reported in environmental data (Fig. 1). These are: size, shape, polymer type, and sorbed environmental contaminants. Although there are other dimensions of microplastics that can drive harm (e.g. presence of biofilm), these four have the most ecotoxicity data and are accessible from environmental monitoring data. Using the available literature, we have ranked the characteristics of these four dimensions from least to most harmful, to inform their hazard. For example, the characteristics of the 'shape' dimension are sphere, fragment, and fiber. Using the framework, a particle-specific hazard value can be calculated, which informs how harmful that microplastic particle is to organisms in the environment. To inform 


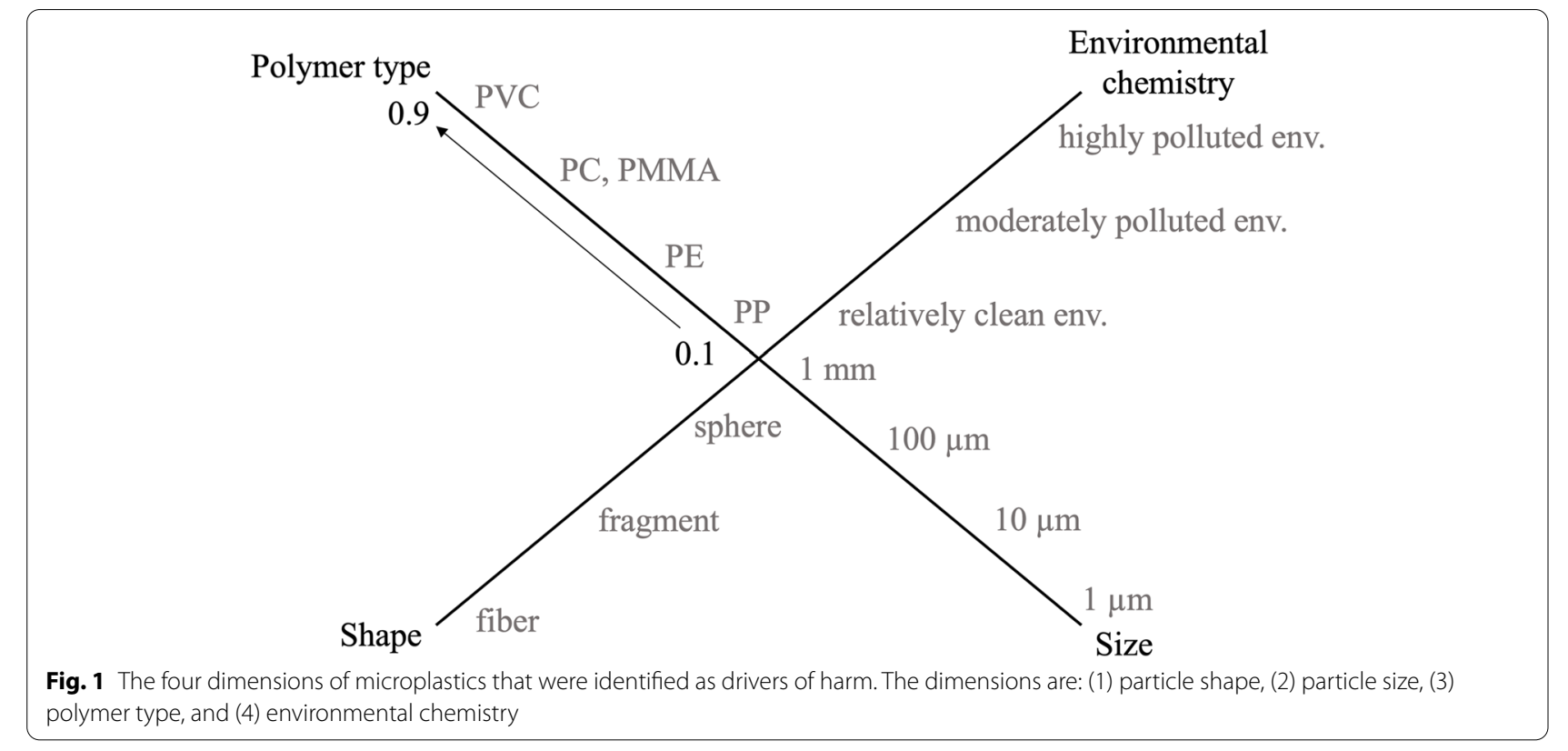

the overall hazard of an environmental sample, the particle-specific hazard values of each unique particle type in an environmental sample can then be summed. Finally, to inform the risk that the sample poses in the environment, the sample's overall hazard can be combined with information about local exposure information (e.g., to achieve a concentration-based risk). The framework we propose here is a skeleton that should be adjusted by a practitioner to include relevant dimensions and by researchers with new and relevant data to increase confidence in the rankings for each dimension. As a next step, we propose a toxicity testing scheme to help improve the proposed rankings, involving strategic testing that identifies the relative toxicity associated with each of the dimensions. The utility of our framework will be to allow decisionmakers to prioritize mitigation strategies for microplastics that are doing the most harm in the environment.

\section{A multidimensional risk assessment framework}

Our framework includes four drivers of stressful action, or 'dimensions', of microplastics, with characteristics that have been ranked from least to most harmful, using the existing body of literature to inform their relative harm (Fig. 1). The dimensions are: 1) particle size, 2) particle shape, 3) polymer type (incorporating polymer and likely additives), and 4) sorbed environmental contaminants. The characteristics of each dimension are ranked in Table 1. For each microplastic particle in an environmental sample, we can then derive a particle-specific hazard value by summing the rankings of each dimension. Note that by summing the rankings of each dimension, our framework assumes that the effects of each dimension are additive. This may not actually be the case, and instead the combined effect of each dimension could be antagonistic or synergistic. With further strategic testing, we can better estimate the rankings and understand whether the effects of each dimension are truly additive, and adapt the framework accordingly. We can then calculate the hazard of an environmental sample by summing the particle-specific hazard values, and combining this with local exposure data to calculate the concentrationbased risk in the environment. Here, we walk through how we ranked the dimensions of microplastics, how to calculate particle-specific hazard, sample hazard, and the risk of a sample. Finally, we demonstrate the utility of our framework using real-world samples collected from San Francisco Bay [17].

\section{Ranking the dimensions of microplastics}

We ranked the characteristics of each of the four dimensions of microplastics from least to most harmful (Table 1; Fig. 1). The rankings were based on the available literature, described in detail below. First, we looked at studies that strategically compared the effects of one dimension while holding the others constant (e.g. [11, $13,15])$. We also looked at patterns noted in reviews synthesizing the effects of microplastics and toxicity tests that investigated a single microplastic type at a time $[9$, $10,16]$. Our rankings are not conclusive - more relevant studies are required to accurately compare the relative harm of each characteristic, as we outline in the future work section. 
Table 1 Ranking the characteristics associated with the four dimensions of microplastics that drive their toxicity. Rankings for polymer type are based on classifications in Lithner et al. [12]. Other dimensions are ranked based on the available literature and the authors' understanding of the relative harm of each characteristic

\begin{tabular}{|c|c|c|c|c|}
\hline Ranking & Size & Shape & Polymer type & Environmental chemistry \\
\hline 0.1 & $>1 \mathrm{~mm}$ & Sphere & PP, PVAc, cellulose & pristine or relatively clean water body \\
\hline \multicolumn{5}{|l|}{0.2} \\
\hline 0.3 & 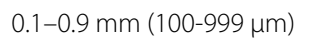 & & PS, LDPE, HDPE, PET & \\
\hline 0.4 & $\begin{array}{l}0.01-0.09 \mathrm{~mm} \\
(10-99 \mu \mathrm{m})\end{array}$ & & & \\
\hline 0.5 & & & Polyamide, EPS & moderately polluted water body \\
\hline 0.6 & & Fragment & & \\
\hline 0.7 & $0.001-0.009 \mathrm{~mm}(1-9 \mu \mathrm{m})$ & & PC, PMMA & \\
\hline \multicolumn{5}{|l|}{0.8} \\
\hline 0.9 & & Fiber & PVC, PUR, ABS, rubber & $\begin{array}{l}\text { highly polluted water body } \\
\text { (e.g. wastewater effluent, highly } \\
\text { populated, industrial, agricultural } \\
\text { areas) }\end{array}$ \\
\hline
\end{tabular}

For particle size, we ranked larger microplastic particles as the least harmful, and small particles as the most harmful. These rankings are based on research that has shown that smaller microplastics are more harmful than larger microplastics due to their increased bioavailability [5], their ability to translocate across membranes and cause further internal damage [18], and their increased surface area to volume ratio [19]. In our framework, large microplastic particles in the size range of $>1 \mathrm{~mm}$ were ranked as the least harmful (ranking 0.1). Intermediately sized microplastic particles, in the size ranges of $0.1-0.9 \mathrm{~mm}$ and $0.01-0.09 \mathrm{~mm}$ were given rankings of 0.3 and 0.4 , respectively. Finally, small particles in the size ranges of $0.001-0.009 \mathrm{~mm}$ were given the highest ranking of 0.7. Although data suggests even smaller particles would have a higher hazard ranking, we have intentionally left nano-sized particles $(<1 \mu \mathrm{m})$ out of our framework since we currently cannot accurately quantify their presence in environmental samples. Moreover, nanosized particles may behave differently than microplastics and may require a separate risk framework. Further research could elucidate whether smaller-sized particles should be included, and whether their rankings would be greater than 0.7 in the framework proposed here. Moreoever, there is also data suggesting that larger particles may be more harmful than smaller particles at smaller concentrations when food dilution is the mechanism of adverse effect [8]. Thus, further work is needed to substantiate our rankings, and to determine whether particle size and toxicity scale linearly, as we assume here.

For particle shape, we ranked spheres as the least harmful, fragments as intermediate, and fibers as the most harmful. These rankings are based on primary research articles, including studies that have tested each particle shape individually and those that have comparatively tested multiple shapes. For instance, fragments and fibers have been shown to be more toxic than spheres to shrimp [15] and Ceriodaphnia [20]. In our framework, we have tentatively ranked spheres as 0.1 , fragments as 0.6 , and fibers as 0.9 . Once again, more research will be needed to substantiate the relative toxicity of each shape, for instance to determine if fibers are actually three steps above fragments, or if they are actually more similar in the relative toxicity. Furthermore, environmental samples are comprised of more than just three particle shapes. More research is needed to determine where shapes such as films, foams, shavings, fiber bundles, etc. fit into this framework.

For polymer type, we ranked the characteristics based on Lithner et al. [12], where polymer types were classified into five hazard categories based on the inherent toxicity of the monomers of which they are made. Here, we ranked polymer types such as polypropylene, PVAc, and cellulose as being the least harmful polymer types, while PVC, PUR, ABS, and rubber were ranked as the most harmful. We spread the five hazard categories provided in Lithner et al. [12] across our 9 hazard rankings. Future research will be needed to substantiate the relative toxicity of each polymer type, including in combination with common additives used to make specific product types.

The environmental chemistry of the particles is based on a gradient of how polluted the surrounding environment is. Particles collected from pristine or relatively clean bodies of water are likely to have a relatively low environmental contaminant load, and thus are given a low ranking. Particles collected from water bodies that are highly polluted, urbanized, industrial and/or agricultural will have a much more harmful environmental 
contaminant load and are thus given the highest ranking. This ranking scheme is a gradient, so each sample can be given a ranking from 0.1 to 0.9 based on how polluted the surrounding environment is. In locations where the data is available, the ranking can be based on water quality data and presence of contaminants (such as legacy contaminants, POPs, heavy metals). Where data is not available, the ranking can be based on proximity to urbanization, industry, and agriculture.

Future iterations of this framework shall be based on more relevant data (and thus be more accurate), and based on dimensions most relevant to the application. For instance, if a decision-maker decided that presence of biofilm was a particularly important driver of risk in their local context, they could easily add biofilm as a dimension in the framework. Moreover, it is important to note that the risk value and subsequent management actions dictated by the framework represent only the current situation. As we know, microplastics are a dynamic contaminant, and the hazard they pose can change with shifting inputs and degradation of particles. Thus, we recommend that this framework be used in an on-going environmental monitoring and risk assessment framework, such that new management actions can be triggered if conditions change. Here, we are providing a starting point and call for more research to build a body of toxicity data suited for informing a risk framework that accounts for the multidimensionality of microplastics.

\section{Determining particle-specific hazard values}

To determine the hazard value associated with a specific microplastic particle, the rankings of each dimension are summed. For example, a spherical, $150 \mu \mathrm{m}$ PS particle purchased directly from a manufacturer would rank as follows: size 0.3 , shape 0.1 , polymer type 0.3 , environmental chemistry 0.1 . Thus, the particle-specific hazard value is 0.8 . In another example, a $150 \mu \mathrm{m}$ fragment of PVC collected from a lake, would rank as follows: size 0.3 , shape 0.1 , polymer type 0.9 , environmental chemistry 0.9 . The particle-specific hazard value of this particle is 2.2. Comparing these two examples demonstrates that the more harmful particle has a larger particle-specific hazard values. Actual environmental samples, however, are comprised of a complex mixture of individual particles of different sizes, shapes, polymer types, and chemical cocktail. For an environmental sample, we can combine the particle-specific hazard values of each unique particle type in the sample to quantify the overall hazard of the sample.

\section{Determining risk for an environmental sample}

Our risk assessment framework can be used to quantify the risk of an environmental sample, provided that a representative subset of the particles in the sample is characterized by size, shape, and chemical ID. First, a particle-specific hazard value is calculated for each particle type (i.e. particles of the same size range, shape, polymer type, and chemical cocktail) as described above. Then, the particle-specific hazard values will be multiplied by the number of particles of that type in the sample. The hazard of the environmental sample can then be calculated by summing the hazard values for each unique particle type. To calculate the risk of the environmental sample, the total hazard score is then divided by the volume (or mass) of the sample. The final risk value will be concentration-based, and is the value that can be used in a risk assessment framework with thresholds set to determine management actions (e.g. Fig. 2).

\section{A case study in San Francisco Bay}

To demonstrate how this risk assessment framework can be used, we ran a case study using three types of samples collected from San Francisco Bay in 2017: a surface water manta trawl, a depth-integrated stream sample taken during the peak of a storm (i.e. stormwater sample), and the final effluent from a wastewater treatment plant. These samples were analyzed for microplastics, and the data from these samples is published in Zhu et al. [17]. See Table $\mathrm{S} 1$ for all data used in the case study, and Table 2 for a summary of the results following our

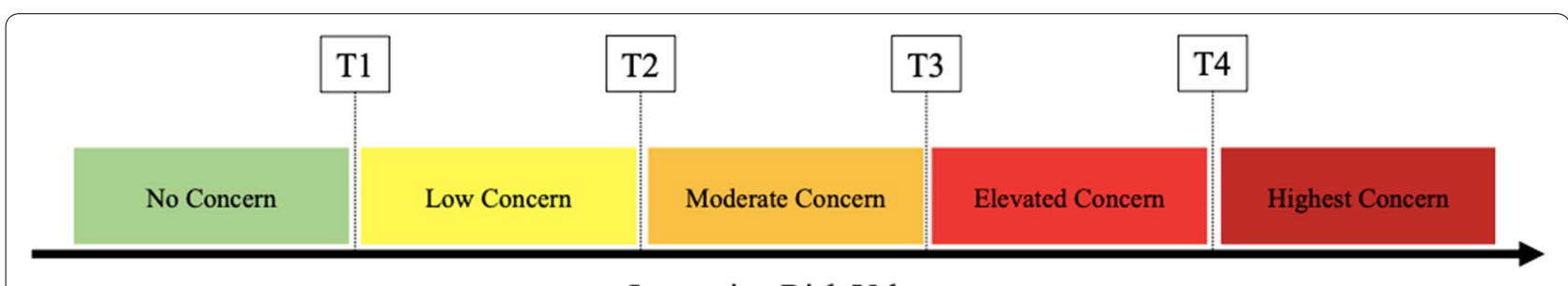

Increasing Risk Values

Fig. 2 An example of a risk management framework that can utilize the framework prposed here. T1,T2, T3 and T4 are proposed threshold values that would trigger a management action relative to different levels of concern 
Table 2 San Francisco Bay case study summary. The sample hazard is based on the type and number of particles. The risk also incorporates the volume concentration in the sample

\begin{tabular}{lllllcc}
\hline $\begin{array}{l}\text { Sample } \\
\text { number }\end{array}$ & Sample type & $\begin{array}{l}\text { Total number of } \\
\text { MPs }\end{array}$ & Sample volume $(L)$ & $\begin{array}{l}\text { Concentration (\# } \\
\text { MP/L) }\end{array}$ & Sample hazard & Sample risk \\
\hline 1 & Stormwater & 1202 & 197 & 6.10 & 2908.6 & 14.76 \\
2 & Manta trawl & 173 & 176,000 & 0.00098 & 406.1 & 0.0023 \\
3 & Wastewater & 77 & 916 & 0.084 & 170.6 & 0.19 \\
\hline
\end{tabular}

framework. In these samples, all suspected microplastic pieces observed were counted and characterized by size, color, and morphology, and a subsample was chemically characterized via spectroscopy or py-GCMS $[17,21]$.

For our risk assessment framework, all particles need to be characterized by size, shape and polymer type. As such, we assumed that the chemical analysis was based off of a representative subsample to extrapolate polymer identifications for all particles within a specific size fraction and morphology. Future application of the risk assessment framework should use datasets where an assumption that a subsample is representative is appropriate. We then used the spectroscopy-corrected dataset to rank each confirmed anthropogenic particle in each sample, and made a choice to not discard the particles categorized as anthropogenic unknown or cellulose that were chemically dyed. These were categorized as anthropogenic cellulose (and thus given a low hazard ranking of 0.1 ) to be conservative. A particle categorized as cellulose using Raman that was natural in color, was considered natural and left out of the calculation.

Using the framework proposed here, we then characterized each type of particle in the sample by summing the rankings for each of their characteristics (Table S1). For example, consider a PET fiber in the 100-999 $\mu \mathrm{m}$ size range from a San Francisco Bay stormwater sample. This particle type was ranked as follows: polymer type 0.3 , shape 0.9 , size 0.3 , environmental chemicals 0.9 . Its particle specific hazard score was thus 2.4. We then multiplied this particle-specific hazard value by the number of particles of that type in the sample. Since there were 94 particles of this type in the sample, our hazard score for this particle type is 225.6. We did this process for each unique particle type in each of the three samples, and then summed all particle-specific hazard scores to calculate the hazard value of the sample (Table S1). The hazard values for the three San Francisco Bay samples were 2908.6 for stormwater, 406.1 for manta trawl, and 170.6 for wastewater (Table 2). Finally, we incorporated local exposure information (i.e., concentration) to calculate the risk of each sample by dividing the sample hazard by the volume of the sample. In our example, the final risk values were 14.8 for the stormwater sample, 0.002 for the surface water sample, and 0.19 for the wastewater sample (Table 2). These results indicate that the stormwater sample has the highest risk in the environment. The high risk value of the stormwater sample is likely being driven by the relatively high concentration of microplastics compared to the other two samples (6 particles per $\mathrm{L}$ in the stormwater sample, 0.001 particles/L in the manta sample, and 0.08 particles/L in the wastewater sample; Table 2) as well as the large amount of tire wear rubber. In this framework, risk will largely be driven by concentration (which is likely most relevant) but will be affected by particle type. For example, a water sample with 100 particles/L of PP microbeads will have a lower risk value than a water sample with 100 particles/L of tire wear rubber. The results from this case study suggest that mitigating microplastics in stormwater runoff is the most efficient use of resources in terms of benefit to the environment. Here, we have presented a case study using environmental samples from the San Francisco Bay. However, our framework could be applied to any other sampling regime where polymer identification is performed for a representative subsample of particles.

\section{Applying the risk value to a risk assessment}

Once complete, the risk assessment framework provides a risk value that incorporates both the amount and diversity of particles in the sample. This allows for more toxic microplastics to have a higher risk at low concentrations, and for less toxic particles to have a higher risk at higher concentrations. Here, toxicity is a function of the particle's shape, size, polymer type, and chemical load. As such, our framework takes into account both the chemical and physical effects of microplastics to assess the risk of an environmental sample. The next step is then to interpret what the risk of the sample means. This interpretation is up to the environmental decision-maker based on their objectives and risk tolerance in the system. Ideally, threshold values would be applied across a management framework whereby certain risk values would trigger specific management actions - e.g., monitoring, source-control, recreational fishing guidelines, etc. (see [8]). These thresholds could be guided by metaanalyses of the existing literature [22], or future toxicity tests designed to better answer these questions relevant to how the multidimensionality informs risk. An example 
of how this may look is in Fig. 2, but should be adapted by managers based on the best available science. Here, our main objective was to introduce a multidimensional framework for assessing the risk of microplastics, thus we do not make suggestions for thresholds or management actions. Rather, we designed a framework that can be adapted as research gaps are filled and management decisions are made.

\section{Future work needed to strengthen the framework}

Here, we have proposed a framework that incorporates the multidimensionality of microplastics to understand their hazard in the context of risk assessment. Our framework is based on the available literature and the authors' understanding of what drives the toxicity of microplastics. However, we recognize that more work is needed to help substantiate and improve our rankings. Research that has the specific aim of understanding the relative toxicity of each dimension of microplastics is needed. Careful consideration must be made towards the design of the study and the question(s) being asked. For instance, in a study that aims to determine which plastic shape is the most harmful, all other dimensions of microplastics (i.e. size, polymer type, chemical cocktail, concentration) must be held constant between treatments (Fig. 3). Further work may also consider the biofilm that attaches to microplastics in the environment, perhaps as another dimension, which is known to increase the ingestion rate of particles [23] and increase their internalization into cells [24] compared to pristine particles. As we fill out the body of toxicity data by investigating individual dimensions of microplastics, the natural next step will be to begin investigating the dimensions as a multiple stressor, where multiple dimensions would be manipulated to investigate interactive effects. Further work should also consider how the toxicity of microplastics is altered by the environment, as temperature and $\mathrm{pH}$ have been shown to modulate the impacts of microplastics to zooplankton [25] and sea urchins [26]. Thus, microplastics are not only a multiple stressor on their own, but also exist in a multiple stressor world. Additional research will help us understand how the dimensions of microplastics interact with each other and with the environment to drive toxicity.

Future experimental work to be used in our framework must also prioritize using standardized testing methodologies and environmentally realistic exposure scenarios. Such standard testing methods have yet to be developed, and may benefit from lessons learned from nanomaterials and colloidal materials. Moreover, they should include standards for quality control. A recent systematic review assessed the quality of studies testing the effects of microplastics,

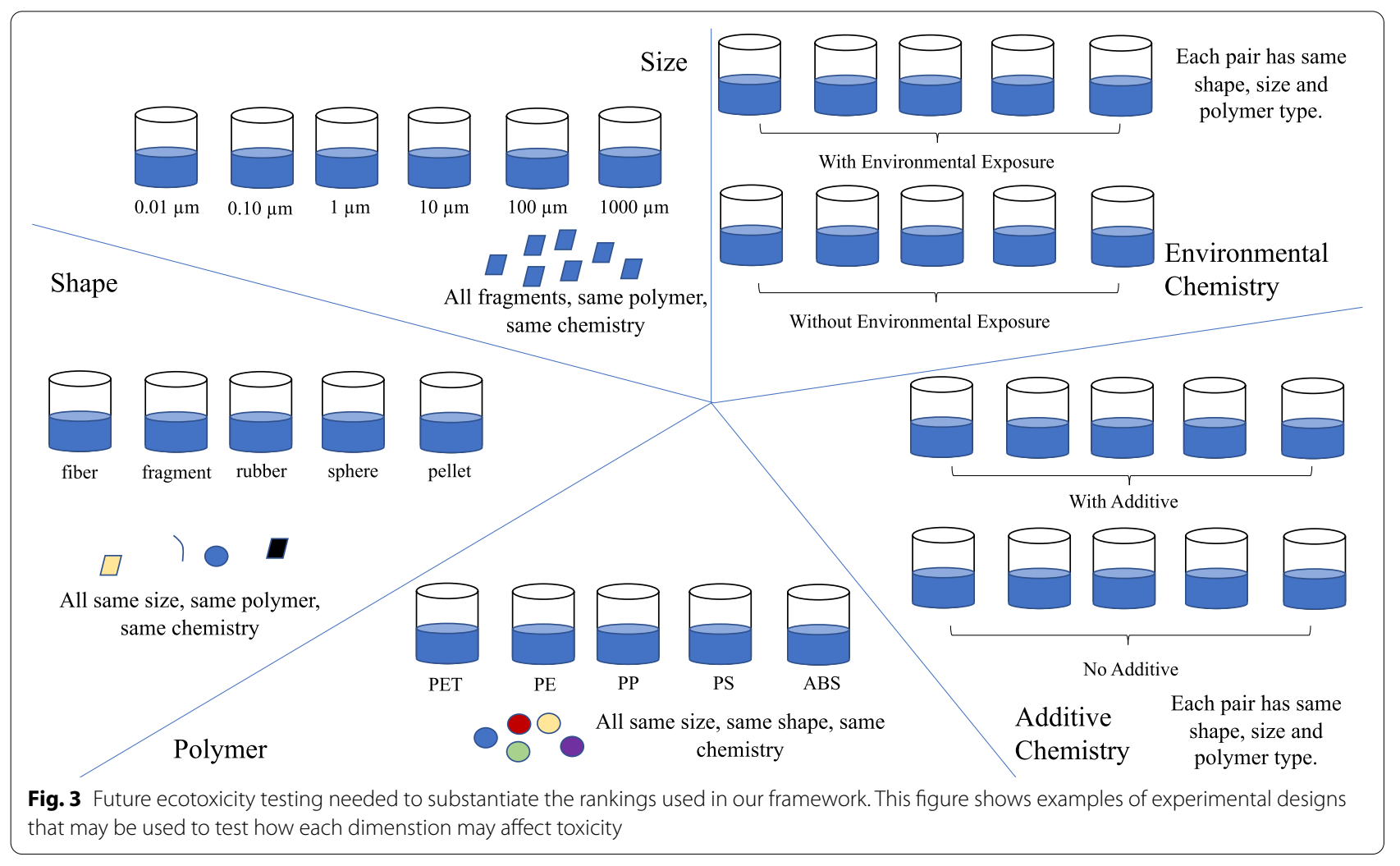


and found an urgent need for better methodologies and quality assurance in microplastics effects testing [27]. Their guidelines include having accurate particle characterization, using environmentally relevant concentrations, and quality assurance protocols [27]. Furthermore, for studies to be informative to ecological risk assessments, they must target endpoints that are ecologically relevant, and use exposure durations that allow chronic effects to be determined. Endpoints such as mortality, growth and development, and reproduction, are especially useful to indicate whether microplastics cause effects at the population level. Here, we have not ranked the data based on a biological endpoint, but in a future iteration where more data is available, we may choose to prioritize an endpoint that is more informative to the objective of the risk assessment.

The toxicity testing scheme that we propose is elaborate, and will likely require harmonization and collaboration within and across research groups. However, we contend that the most efficient way to understand the 'effects of microplastics' is by investigating how the dimensions of particles influence their toxicity, rather than by testing each and every particle individually.

\section{Conclusions}

We have proposed a framework for evaluating the multidimensional risks of microplastics in the environment. We identified four dimensions of a microplastic particle that can drive risk (i.e. shape, size, polymer type, environmental chemistry). We specifically picked dimensions that are both well-studied and commonly reported in environmental data. We show how these dimensions can inform and generate a particle-specific hazard value for microplastic. The resulting particle-specific hazard values can be combined to calculate the hazard of an environmental sample. Finally, by incorporating the concentration of the microplastics per volume of sample, we show how to calculate a risk value for the environmental sample of interest. The risk score calculated using our framework can be used in a risk assessment. The risk score can then be interpreted by environmental decision-makers using a management framework that sets threshold values which trigger management decisions, as shown in Fig. 2. Finally, we propose a toxicity testing scheme where experiments are carefully designed to answer questions about the dimensions of microplastics that drive their toxicity. In addition to strategic experimental design, these experiments must prioritize using the best methodologies and environmentally realistic exposure scenarios. Such experiments are necessary to increase the accuracy of this framework for application. Combined, this new framework takes into account the multidimensionality of microplastics and will allow decision-makers to regulate the types and sources of microplastics that are causing the most harm in the environment.

\section{Abbreviations}

PE: Polyethylene; LDPE: Low density polyethylene; HDPE: High density polyethyelen; PP: Polypropylene; PVC: Polyvinyl chloride; PS: Polystyrene; PVAC: Polyvinyl acetate; PET: Polyethylene terephthalate; EPS: Expanded polystyrene; PC: Polycarbonate; PMMA: Polymethyl methacrylate; PUR: Polyurethane; ABS: Acylonitrate butadiene styrene.

\section{Supplementary Information}

The online version contains supplementary material available at https://doi. org/10.1186/s43591-022-00028-0.

\section{Additional file 1.}

\section{Acknowledgements}

The idea to create a multidimensional risk framework for microplastics was sparked by a discussion in a CEE Lab meeting (hosted by Dr. M. Rennie) about contaminants having multiple modes of stressful action. We would also like to thank the two anonymous reviewers whose comments improved our manuscript.

\section{Peer review}

In keeping with research integrity principles, all SCCWRP workshop articles coauthored by Dr. Steve Weisberg, Dr. Albert Koelmans, and other Microplastics and Nanoplastics editors were handled by independent editors of the journal.

\section{Authors' contributions}

$\mathrm{KB}$ and CMR conceptualized and wrote the manuscript. The author(s) read and approved the final manuscript.

\section{Funding}

KB was funded by Ontario Graduate Scholarships during the writing of this manuscript.

\section{Availability of data and materials}

All data analysed in this manuscript are included in the published article and its supplementary information files.

\section{Declarations}

\section{Competing interests}

The authors declare no conflicts of interest.

Received: 25 October 2021 Accepted: 13 January 2022

Published: 15 February 2022

\section{References}

1. Daughton CG. "Emerging" chemicals as pollutants in the environment: a 21st century perspective. Renew Resour J. 2005;23(4):6-23

2. Rochman CM, Brookson C, Bikker J, Djuric N, Earn A, Bucci K, et al. Rethinking microplastics as a diverse contaminant suite. Environ Toxicol Chem. 2019;38(4):703-11.

3. Rochman CM. Plastics and priority pollutants: a multiple stressor in aquatic habitats. Environ Sci Technol. 2013;47(6):2439-40.

4. Teuten EL, Saquing JM, Knappe DRU, Barlaz MA, Jonsson S, Björn A, et al. Transport and release of chemicals from plastics to the environment and to wildlife. Phil Trans R Soc B. 2009;364(1526):2027-45.

5. Wright SL, Thompson RC, Galloway TS. The physical impacts of microplastics on marine organisms: a review. Environ Pollut. 2013;178:483-92.

6. Koelmans AA, Redondo Hasselerharm PE, Mohamed Nor NH, Kooi $M$. Solving the non-alignment of methods and approaches used in microplastic research in order to consistently characterize risk. Environ Sci Technol. 2020;54(19):12307-15 Sep 4;acs.est.0c02982.

7. Everaert G, De Rijcke M, Lonneville B, Janssen CR, Backhaus T, Mees J, et al. Risks of floating microplastic in the global ocean. Environ Pollut. 2020;267:115499. 
8. Mehinto AC, Coffin S, Koelmans A A, Brander S, Wagner M, ThorntonHampton L, et al. Risk-based management framework for microplastics in aquatic ecosystems. Microplastics and Nanoplastics. 2021; this issue.

9. Foley CJ, Feiner ZS, Malinich TD, Höök TO. A meta-analysis of the effects of exposure to microplastics on fish and aquatic invertebrates. Sci Total Environ. 2018;631-632:550-9.

10. Bucci K, Tulio M, Rochman CM. What is known and unknown about the effects of plastic pollution: a meta-analysis and systematic review. Ecological Applications. 2020:30(2):e02044.

11. Abarghouei S, Hedayati A, Raeisi M, Hadavand BS, Rezaei H, AbedElmdoust A. Size-dependent effects of microplastic on uptake, immune system, related gene expression and histopathology of goldfish (Carassius auratus). Chemosphere. 2021;276:129977.

12. Lithner D, Larsson A, Dave G. Environmental and health hazard ranking and assessment of plastic polymers based on chemical composition. Sci Total Environ. 2011;409(18):3309-24.

13. Lithner D, Nordensvan I, Dave G. Comparative acute toxicity of leachates from plastic products made of polypropylene, polyethylene, PVC, acrylonitrile-butadiene-styrene, and epoxy to daphnia magna. Environ Sci Pollut Res. 2012:19:1763-72.

14. Rochman CM, Kurobe T, Flores I, Teh SJ. Early warning signs of endocrine disruption in adult fish from the ingestion of polyethylene with and without sorbed chemical pollutants from the marine environment. Sci Total Environ. 2014;493:656-61.

15. Gray AD, Weinstein JE. Size- and shape- dependent effects of microplastic particles on adult daggerblade grass shrimp (Palaemonetes pugio). Environ Toxicol Chem. 2017;36(11):3074-80.

16. Paul-Pont I, Tallec K, Gonzalez-Fernandez C, Lambert C, Vincent D, Mazurais $\mathrm{D}$, et al. Constraints and priorities for conducting experimental exposures of marine organisms to microplastics. Front Mar Sci. 2018:5(July):1-22

17. Zhu X, Munno K, Grbic J, Werbowski LM, Bikker J, Ho A, et al. Holistic assessment of microplastics and other anthropogenic microdebris in an urban bay sheds light on their sources and fate. ACS EST Water. 2021;1(6):1401-10.

18. Browne M, Dissanayake A, Galloway T, Lowe D, Thompson R. Ingested microscopic plastic translocates to the circulatory system of the mussel, mytilus edulis (L.). Environ Sci Technol. 2008;42:5026-31.

19. Goedecke C, Stollin UM, Hering S, Richter J, Piechotta C, Paul A, et al. A first pilot study on the sorption of environmental pollutants on various microplastic materials. J Environ Anal Chem [Internet]. 2017 [cited 2021 Oct 6];04(01). Available from: https://www.omicsgroup.org/journals/afirst-pilot-study-on-the-sorption-of-environmental-pollutants-on-vario usmicroplastic-materials-2380-2391-1000191.php?aid=87741

20. Ziajahromi S, Kumar A, Neale PA, Leusch FDL. Impact of microplastic beads and fibers on waterflea (ceriodaphnia dubia) survival, growth, and reproduction: implications of single and mixture exposures. Environ Sci Technol. 2017;51(22):13397-406.

21. Werbowski LM, Gilbreath AN, Munno K, Zhu X, Grbic J, Wu T, et al. Urban stormwater runoff: a major pathway for anthropogenic particles, black rubbery fragments, and other types of microplastics to urban receiving waters. ACS EST Water. 2021;1 (6):1420-8.

22. Thornton-Hampton L, Lowman H, Coffin S, Darin E, De Frond H, Hermabessiere $L$, et al. A living tool for the continued exploration of microplastic toxicity. Microplastics and Nanoplastics. 2021; this issue.

23. Vroom RJE, Koelmans AA, Besseling E, Halsband C. Aging of microplastics promotes their ingestion by marine zooplankton. Environ Pollut. 2017;231:987-96

24. Ramsperger A, Narayana V, Gross W, Mohanraj J, Thelakkat M, Greiner A, et al. Environmental exposure enhances the internalization of microplastic particles into cells. Sci Adv. 2020;9(6):1-9.

25. Lyu K, Cao C, Li D, Akbar S, Yang Z. The thermal regime modifies the response of aquatic keystone species daphnia to microplastics: evidence from population fitness, accumulation, histopathological analysis and candidate gene expression. Sci Total Environ. 2021;783:147154.

26. Bertucci Iا I, Bellas J. Combined effect of microplastics and global warming factors on early growth and development of the sea urchin (paracentrotus lividus). Sci Total Environ. 2021;782:146888.

27. de Ruijter VN, Redondo-Hasselerharm PE, Gouin T, Koelmans AA. Quality criteria for microplastic effect studies in the context of risk assessment: a critical review. Environ Sci Technol. 2020;54(19):11692-705.

\section{Submit your manuscript to a SpringerOpen ${ }^{\odot}$ journal and benefit from:}

- Convenient online submission

- Rigorous peer review

- Open access: articles freely available online

- High visibility within the field

- Retaining the copyright to your article

Submit your next manuscript at $\boldsymbol{\nabla}$ springeropen.com 